\title{
A TRIP TO CENTRAL PARK
}

\section{Love at first sight?}

\section{BY MICHAEL ADAM ROBSON}

$\mathrm{I}$ $t$ felt a signal deep in a lower layer of space, a pattern that indicated intelligence. Curious, it built a child process in the layer's native physics and sent it to investigate.

This layer of space was constraining in its dimension, confusing in its uncertainty and linearity. A hot blob of plasma dominated the area where it had spawned, but there was no discernible pattern in the energy that radiated from it. No, the signal was leaking from a nearby child mass, a relatively small, cold glob of matter.

The mass was overgrown with the kind of replicating chemical processes that were common in this layer of space. These particular processes, as sometimes happened, encoded a kind of simple intelligence.

There was evidence of industry, ordered structures growing out of the chaos, an evolving framework of technology. The mass was working to attain sentience. A rare event, and it would be there to observe that happen!

It chose a rectangular expanse of chemical life fenced in on four sides by rows of tall, rectangular structures, and it discreetly spawned a small child process there, building it in the native chemistry.

A cluster of life forms stood before it. Cautious, it slithered to the closest one, absorbing the light it reflected, inhaling the carbon-based molecules it exuded. When the creature didn't react to its approach, it wrapped itself around the body, oozing into the rough texture. It flexed and snapped off a limb, tasting the creature's chemistry.

The creature seemed to subsist through a simple reaction, harvesting matter and electromagnetic energy, and converting it to chemical stores. Energy from the large plasma ball was probably the main driving force behind the chemical processes on this smaller mass.

Individually, this creature seemed far too simple to have any kind of intelligence. Perhaps they worked collectively.

It detected motion and noticed for the first time, hiding behind and among the life forms, a herd of smaller creatures - possibly their replicated offspring. These creatures were more mobile, and they wobbled away from it as it turned. Several held up rectangular objects that suggested crafting - a display of art or technology?

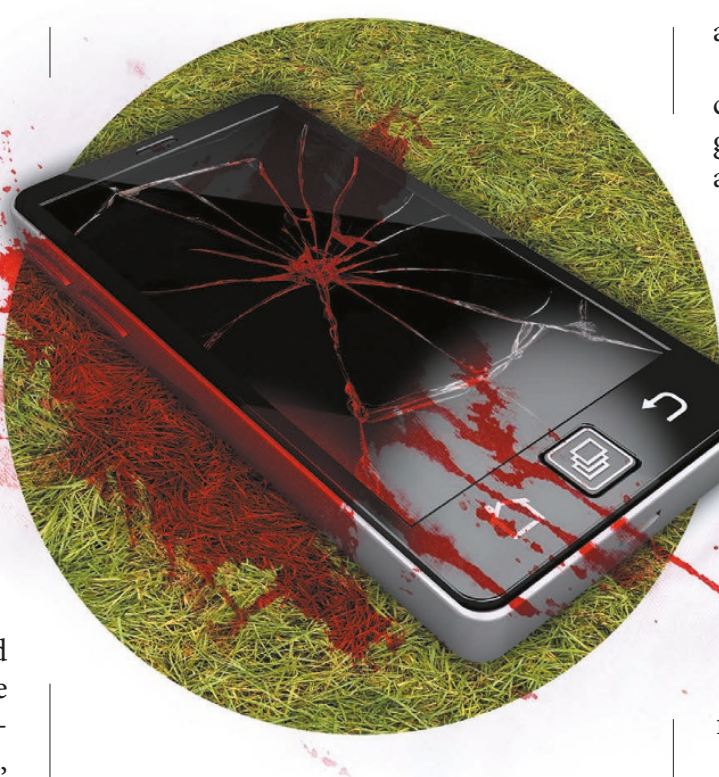

The rectangle seemed to be significant to this culture, perhaps a fetish linked to their replication processes; the creatures themselves had blobby, vaguely symmetrical shapes.

The objects emitted slow electromagnetic waves, and these waves encoded more complex patterns - they were connected to the signal it had detected! Could these simple machines be the dominant intelligence here? It transmitted the waves back at them, with no obvious response.

It slid nearer, and again the creatures wobbled away, perhaps distressed by its alien appearance. It stopped, and condensed its mass into a bulbous shape roughly similar to theirs.

Vibrations rippled from the creatures, the air serving as a channel of information between them. They were communicating, but the message was lost on it. Again, it attempted to parrot the vibrations back, and this time it seemed to elicit a reaction.

Before long, more curious creatures joined the herd. They were carried by larger machines, and themselves carried other machines of different shapes and sizes - another exhibition of their primitive technology.

A few of these creatures marked a large space around it with a strip of material, a $\rightarrow$ NATURE.COM

Follow Futures: @NatureFutures $f$ go.nature.com/mtoodm rectangle within a rectangle. It understood this act was a ritual meant to keep it separated from the herd, and it respectfully remained inside the lines.

More and more of the creatures arrived, crowding around the borders of the rectangle, pointing their machines, rippling the air with their vibrations.

And then the vibrations stopped.

The herd parted, and one of the creatures approached. It crossed the ritual border, and slowly tottered its way over.

For a while, the creature just balanced on two limbs, observing it. Then it extended a third limb. An offering.

As with the first creature it had encountered, it pulled off the branch and sampled its chemistry. A hot, iron-rich fluid spattered over it.

The de-limbed creature wobbled on the ground, spewing fluid and producing powerful vibrations that were taken up by the herd. A strong response. Perhaps it had triggered a replication process. Did the creature want to exchange chemical material with it? It politely chose not to expel the material it had been sprayed with, and instead sloughed some of its own mass off on top of the thing.

Meanwhile, creatures in the back of the herd had raised the large machines they'd been carrying. The air ripped with clattering vibrations, and it was pelted with a collection of small, high-velocity objects. Was this another display of technology? No, the objects had been directed at it, this was another attempt at communication, vibration coupled with a tactile channel. It mimicked the clatter, and pelted the herd with its own barrage of high-velocity mass.

All around it, creatures wobbled on the ground and expelled fluid and vibrations. The grand display of primitive replication was disturbing, but at the same time it was also amusing. After all, so many first contacts ended in misunderstanding, and even aggression ... wasn't this amorous encounter preferable?

Some of the creatures hobbled off, their primitive appetites satisfied, but others came to take their place, machines converging from the ground and the air. Encouraged, it emitted a powerful clatter and went to greet them.

Michael Adam Robson is an engineer and artist living in Vancouver, British Columbia. This is his second story for Futures. 